\title{
Effects of the inclination of context lines on perception of the Ponzo illusion by pigeons
}

\author{
KAZUO FUJITA \\ Kyoto University, Inuyama, Aichi, Japan \\ and \\ DONALD S. BLOUGH and PATRICIA M. BLOUGH \\ Brown University, Providence, Rhode Island
}

\begin{abstract}
Pigeons discriminated the length of a bar located between two context lines. Responses to one key were reinforced when the bar was longer than a predetermined length, and those to the other key were reinforced when the bar was shorter. The inclination of the context lines was systematically varied from $54.6^{\circ}$ (converging upward) to $125.4^{\circ}$ (converging downward). Five out of 6 subjects tended to report "long" when the bars were located near the apex of the context lines, regardless of whether the context lines were oriented upward or downward. The magnitude of the illusion varied almost linearly with the ratio of the length of the stimulus bar to the gap between the bar and the context lines. This relationship held equally for upward-and downwardconverging context lines.
\end{abstract}

When we observe two identical parallel bars located between two lines that make an inverted $V$ shape, the bar nearer the apex of the lines looks longer. This phenomenon has been called the Ponzo illusion.

Recently, Fujita, Blough, and Blough (1991) demonstrated that pigeons also perceive this illusion. For example, pigeons had difficulty in learning a simultaneous discrimination between the lengths of two bars located between converging context lines only when the Ponzo illusion could decrease the perceived difference in bar length (Experiment 1). Also, when pigeons were trained to report the length of a single bar located between converging lines, they tended to report "long" as the bar approached the apex of the converging context lines (Experiments 2 and 3).

In a comparative study of the illusion, it is important not simply to demonstrate illusory perception but also to examine the effects of contextual parameters. If humans and pigeons show similar effects of context, for example, a homologous relationship is suggested. In our previous report, we actually addressed effects of additional linear perspective on the magnitude of the illusion by using context displays designed to vary perspective strength: eight lines that converged to one point versus eight that did not. We found no effect of such linear perspective

\footnotetext{
This work was conducted during the first author's stay at Brown University as a Visiting Scholar, sponsored by the Fellowship Program for Japanese Scholars and Researchers to Study Abroad from the Japan Ministry of Education, Science, and Culture. The study was also supported by NSF Grant BNS 88-19876 to the second and third authors. Correspondence may be addressed to Kazuo Fujita, Department of Psychology, Primate Research Institute, Kyoto University, Kanrin, Inuyama, Aichi, 484, Japan (e-mail: fujita@pri.kyoto-u.ac.jp).
}

(Experiment 3). This result was consistent with human data (Fineman \& Carlson, 1973; Newman \& Newman, 1974).

The present experiment addressed the effect of the inclination and orientation of the context lines on the magnitude of this illusion in pigeons. The inclination ranged from $54.6^{\circ}$ to $125.4^{\circ}$. The orientation was either upward or downward. In humans, the magnitude of the Ponzo illusion changes with the inclination of context lines, reaching a maximum at an inclination of about $30^{\circ}$ to $60^{\circ}$ (e.g., Pressey, 1974b; Pressey, Butchard, \& Scrivner, 1971). It also changes with the rotation of the whole illusory figure, although this effect is not large for line drawings (Brislin, 1974; Leibowitz, Brislin, Perlmutter, \& Hennessy, 1969).

\section{METHOD}

\section{Subjects}

The subjects were 6 male Carneaux pigeons (Columba livia) housed in separate cages. Their body weights were kept at approximately $80 \%$ of their free-feeding weights. Three of the birds (Birds 544,942 , and 936) had served as the subjects in the previous study (Fujita et al., 1991). The remaining birds were experimentally naive.

\section{Apparatus}

The apparatus was the same as that used in the previous study (Fujita et al., 1991). Three boxes $(31.5 \mathrm{~cm}$ wide, $36 \mathrm{~cm}$ high, and $30.5 \mathrm{~cm}$ deep) were located in a dark room. The front wall of each box had a rectangular opening ( $11 \mathrm{~cm}$ wide and $8.5 \mathrm{~cm}$ high). Its lower edge was $18.5 \mathrm{~cm}$ above the floor of the box. A 5-in. color TV (Rhapsody TV-670) was mounted $2.5 \mathrm{~cm}$ behind the opening. An infrared-beam response detector (Carol Touch) was located in front of the TV. Another opening, $13.5 \mathrm{~cm}$ below the TV opening, provided access to a food magazine. The magazine could be illuminated by a 3-W bulb. Personal computers (Atari 800 ) presented graphic patterns on the TV as stimuli, controlled the equipment, 
and recorded the data. White noise was used to mask extraneous sound.

\section{Stimuli}

Black graphic patterns on a white background on the TV were used. Pattern sizes are given below in terms of the number of graphic dots, or pixels, where appropriate, as well as the actual lengths on the TV in millimeters. On the TV, lines composed of 100 pixels measured $32 \mathrm{~mm}$ horizontally and $37 \mathrm{~mm}$ vertically.

Examples of the stimulus patterns appear in Figure 1. All the patterns had a horizontal bar at the center of the TV screen. The bars were either $3.2,3.8,4.5,5.1,5.8$, or $6.4 \mathrm{~mm}(10,12,14$, 16,18 , or 20 pixels, respectively) long and $1.1 \mathrm{~mm}$ ( 3 pixels) thick. Two context lines appeared with the horizontal bar. The context lines were $.6 \mathrm{~mm}$ ( 2 pixels) thick and $23.7 \mathrm{~mm}$ (64 pixels) long vertically. The inclination of the left context line was either $54.6^{\circ}$, $74.0^{\circ}, 86.4^{\circ}, 90.0^{\circ}, 93.6^{\circ}, 106.0^{\circ}$, or $125.4^{\circ}$ on the screen. (These values were obtained by setting the tangent of the line to $+1,+2$, +4 , infinite, $-4,-2$, and -1 , in terms of pixels; that is, go one right and one up, one right and two up, and so forth.) These values were chosen to optimize the smoothness of the graphics. All pairs of context lines were separated by $21.1 \mathrm{~mm}$ (66 pixels) at their midpoints.

The context lines appeared in one of three vertical locations: high, middle, or low. For the middle context, the midpoints of the lines were level with the stimulus bar. For the high context lines, the midpoints were $7.4 \mathrm{~mm}$ ( 20 pixels) higher than the bar. For the low context lines, the midpoints were $7.4 \mathrm{~mm}$ lower than the bar (see Figure 1).

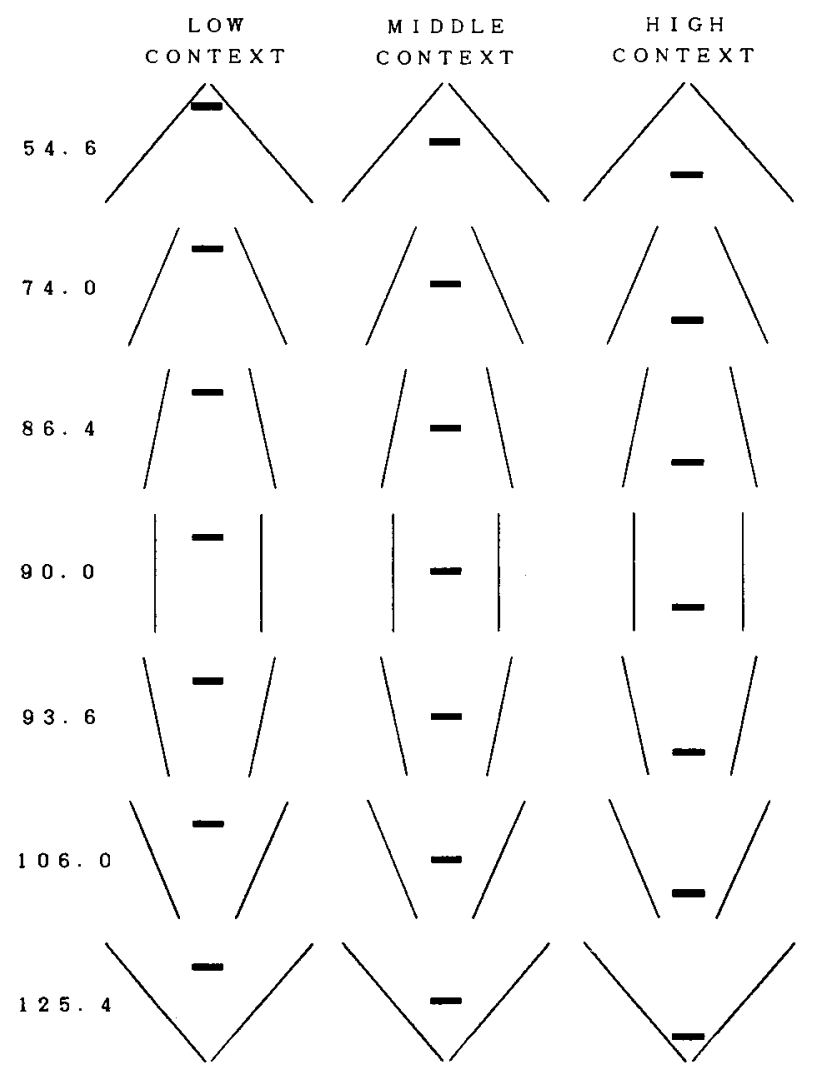

Figure 1. Examples of the stimulus patterns.

\section{Procedure}

The pigeons' task was to peck at one of two locations, depending on the length of the horizontal bar. After intertrial intervals of $5 \mathrm{sec}$, a stimulus pattern appeared with the stimulus bar at the center of the TV. After five pecks at the bar, the bar remained visible and forms marking two choice locations appeared at the bottom two corners of the TV. The left location was marked by an open rectangle of $5.9 \times 6.4 \mathrm{~mm}(16 \times 20$ pixels $)$, and the right location was marked by a filled rectangle of the same size. These choice locations were designated as "long-" or "short-" bar responses, counterbalanced across subjects.

A single peck at the appropriate location was correct and was reinforced either by raising the food magazine accompanied by a flash of the magazine light (food reinforcement) or by the flash of the magazine light alone (conditioned reinforcement). The percentage of food reinforcement for correct choices varied from $100 \%$ to $12.8 \%$, depending upon the phase and the subject. Conditioned reinforcement was given on the remaining correct trials. The duration of food reinforcement was also varied to maintain constant running weights and ranged from 0.9 to $3 \mathrm{sec}$. A single peck at the inappropriate location was an error and was followed by a 5sec darkening of the TV screen. The same trial was then repeated after the error (i.e., a correction method was used).

The three naive pigeons were first autoshaped to peck at a small black square on the TV screen. Then they were trained and tested in the Phase 1 to Phase 4 sequence described below. The three experienced pigeons participated only in Phases 3 and 4 .

Phase 1: Preliminary discrimination of bar length for naive birds. The stimuli were horizontal bars without context lines. The bars were the shortest $(3.2 \mathrm{~mm})$ and the longest $(6.4 \mathrm{~mm})$ of the six. Pecks at the "long" location were correct for 6.4-mm bars, and those at the "short" location for 3.2-mm bars. Percentage of food reinforcement started at $100 \%$ and was gradually decreased to $20 \%$ for Pigeon 4058 , to $25 \%$ for Pigeon 4073 , and to $50 \%$ for Pigeon 2289. The number of trials was initially 96 per session and was increased to 576 by the end of this phase. This phase continued until the subjects performed at more than $85 \%$ correct for two consecutive sessions.

Phase 2: Preliminary discrimination of bars with context lines for naive birds. Context lines were next added to the display in the middle position. The inclination of the context lines was $93.6^{\circ}$ for Pigeon 4058, 74.0 $0^{\circ}$ for Pigeon 4073, and $54.6^{\circ}$ for Pigeon 2289; these were the inclinations used in the first test of the following phase (Phase 3 ) for each subject. The bars were the same as in Phase $1(3.2 \mathrm{~mm}$ and $6.4 \mathrm{~mm})$. The probability of food reinforcement and the number of trials per session were also the same as those at the end of Phase 1 . Phase 2 ended when the birds again chose correctly in $85 \%$ of all trials for two consecutive sessions.

Phase 3: Baseline discrimination of bars with middle context lines. In this phase, the pigeons were trained on the "long" versus "short" discrimination using bars of all six lengths with context lines in the middle position. Responses to the "short" location were reinforced when the bar was either $3.2,3.8$, or $4.5 \mathrm{~mm}$ long, and responses to the "long" location were reinforced when the bar was either $5.1,5.8$, or $6.4 \mathrm{~mm}$ long. All six lengths appeared equally often in randomized order within each session. One inclination of the context lines was chosen for each bird. Percentage of food reinforcement was $20 \%, 20 \%, 25 \%, 20 \%, 25 \%$, and $50 \%$ for Pigeons $544,942,936,4058,4073$, and 2289 , respectively. The number of trials was 576 per session, and at least five sessions were run. After this minimum number of sessions, training continued until (1) overall accuracy was more than $75 \%$ correct, (2) accuracies for the longest and the shortest bars were greater than $80 \%$, and (3) those for two intermediate bars were greater than $40 \%$, for two consecutive sessions. 
Phase 4: Test with high and low context lines. Performances with high and low context lines were tested in Phase 4. Among the 576 trials per session, high and low context lines appeared on 72 trials each (test trials), and bars of all six lengths appeared equally often per context condition. This yielded 12 trials for each combination of bar length and context. Middle context lines appeared on the remaining 432 trials (baseline trials). On these baseline trials, responses were reinforced according to the contingencies described for Phase 3. On test trials, responses were reinforced regardless of the location of the peck, "long" or "short." The probability of food reinforcement was decreased to $80 \%$ of that used in Phase 3. Each test was conducted for two sessions, separated by series of baseline sessions as in Phase 3. At least three baseline sessions were run in between each test session and were continued until each bird again satisfied the criterion described above.

The baseline and test sequence was repeated seven times, once for each of the seven different inclinations of context lines. In other words, only one inclination of the context line was presented on each two-session test, but over tests all inclinations were presented in an order counterbalanced across subjects.

We predicted that if pigeons see the Ponzo illusion, the proportion of "long"' reports should increase as the stimulus bar approaches the apex of the line contexts in testing.

\section{RESULTS}

The naive pigeons $(4058,4073$, and 2289$)$ mastered Phase 1 in 29, 24, and 33 sessions, respectively. All of these subjects mastered Phase 2 in 3 sessions.

In Phases 3 and 4, 5 birds yielded consistent shifts of "long" and "short" reports depending on context position; one bird (4073) did not. For context lines of $74.0^{\circ}$, the latter bird showed a bias supporting the Ponzo illusion, but the tendency reversed for the $54.6^{\circ}$ lines, and there was very little bias for the other five inclinations.
This bird apparently learned something different than the other birds, so its data were excluded from the following analyses.

Figure 2 shows the proportion of "long" responses as a function of bar length averaged for the 5 remaining birds. The top left panel shows the data for the inclination angle of $54.6^{\circ}$ (converging upward), and the top right panel is for $125.4^{\circ}$ (converging downward). The other panels are arranged in order by angle; the middle panel is for $90^{\circ}$. Solid lines with squares represent data from high context patterns, dotted lines with asterisks are from middle context patterns, and broken lines with triangles are from low context patterns.

For the two extreme inclinations (top left and top right), the pigeons showed a consistent tendency to report "long" more often for the patterns in which the bars were near the apex of the context lines. The pigeons showed weaker biases of similar tendency for the contexts involving intermediate inclination angles. No consistent bias appeared at $90^{\circ}$. The patterns of bias for upward-converging contexts (left graphs) closely mirrored those for downwardconverging contexts (right graphs).

Because the variation of the context inclination correlated with the variation of the gap between the stimulus bar and the context lines, it was possible for the pigeons to respond on the basis of the gap size instead of bar length. The top panel of Figure 3 shows the percentages of "long" responses plotted as a function of the gap size (see the pattern in the bottom panel) in order to determine if this variable might have affected the pigeons' choices. Squares are from high context, asterisks are from middle context, and triangles are from low context. The

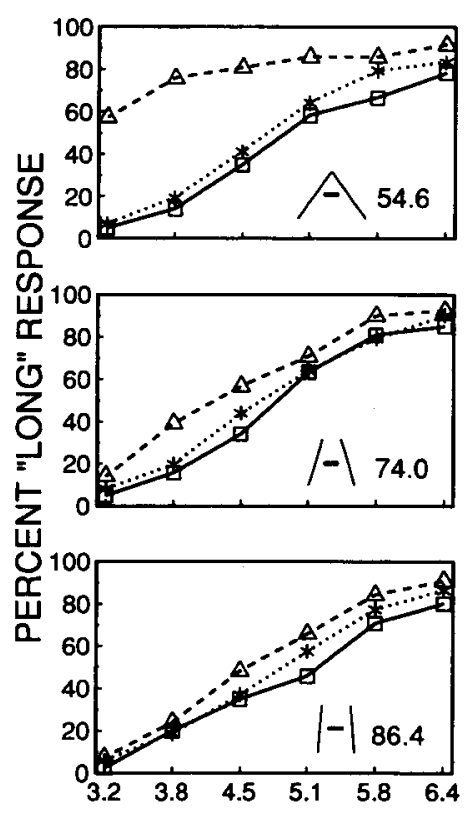

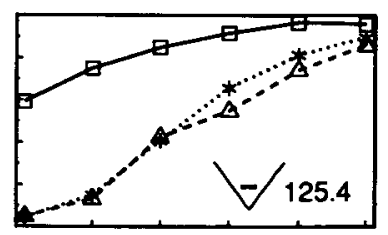
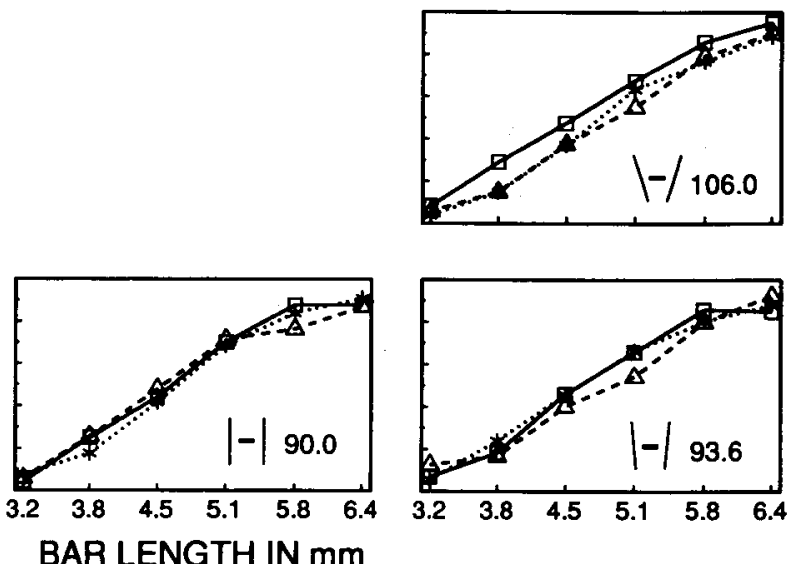

Figure 2. Percentages of "long" responses as a function of bar length for each of the seven inclinations of context lines. The data are averaged for the 5 successful birds. 


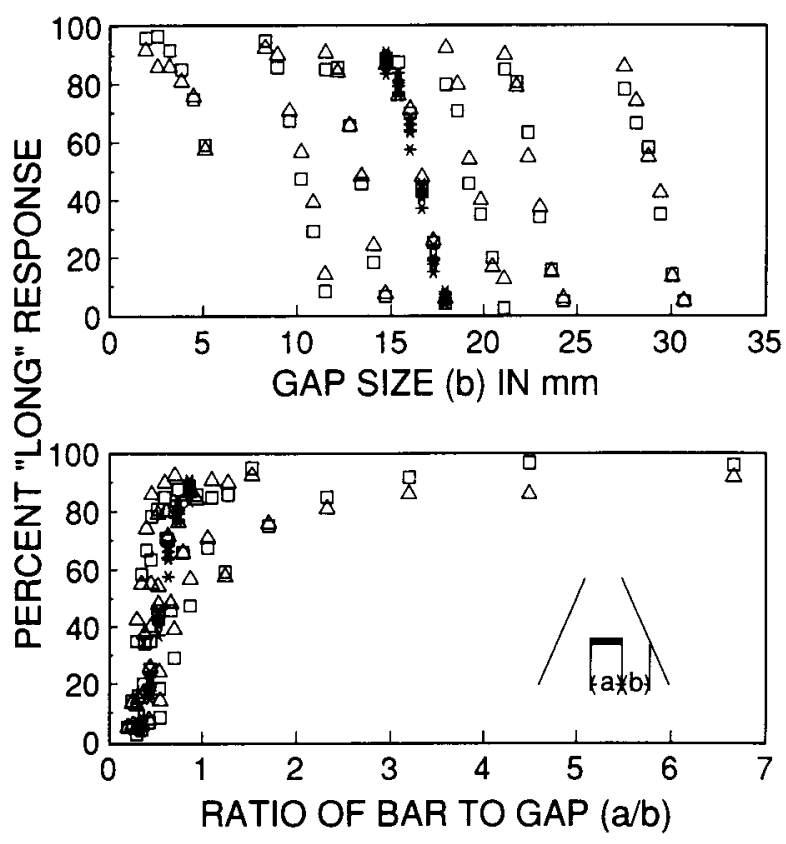

口HIGH CONTEXT * MIDDLE CONTEXT $\triangle$ LOW CONTEXT

Figure 3. Percentages of "long" responses as a function of size of the gap (b) between the bar and the context lines (top panel) and of the ratio of the bar length to the gap ( $a / b$; bottom panel). The data are averaged over the 5 birds showing the illusory effect.

individual data points are the averaged performances derived from each combination of bar length, line orientation, and context. This panel shows that there is no obvious relationship between gap size and the percentages of "long" responses. Clearly, gap size cannot account for the pigeons' performances for any type of context.

In the bottom panei of Figure 3, the same percentages of "long" responses are replotted as a function of the ratios of bar length to gap size (see the pattern in the graph). The symbols are the same as in the top panel. Here, there is a good correlation between the proportion of "long" responses and this independent variable.

For each of the 5 birds, perceived length of bars was calculated in terms of the shift of the point of subjective equality (PSE). First, percentages of "long" choice responses were converted to $z$ scores. Linear regressions were then calculated for each of the three context locations, low, middle, and high. The PSEs were the interpolated values at $z=0$ (50\% "long" choices). The relative perceived length of bars in low and high contexts was taken as the ratio of the PSEs for the middle context condition to those for the low and high conditions, respectively. For example, if PSEs were 3, 5, and $6 \mathrm{~mm}$ for low, middle, and high contexts for one particular inclination, then the relative perceived length of bars in low context lines would be $5 / 3(166.7 \%)$ and that of bars in high context lines would be $5 / 6(83.3 \%)$.
Figure 4 shows the relative perceived length of bars averaged for the 5 successful birds as a function of the inclination of the context lines. The solid line with squares represents high context conditions, and the dotted line with asterisks represents low context conditions. For the high context condition, the perceived length gradually increased with increasing inclination of the context lines from $54.6^{\circ}$ to $106.0^{\circ}$, and showed a sharp jump at $125.4^{\circ}$. The results from the low context condition were almost the mirror image of those from the high context condition. Individual differences in perceived length were quite large, particularly for the two extreme conditions, although all 5 birds overestimated the bar length. The perceived lengths for high context of $125.4^{\circ}$ were 1.475 , 2.903, 1.344, 1.334, and 2.771 for Pigeons 544, 942, 936,4058 , and 2289 , respectively, and those for low context of $54.6^{\circ}$ were $2.151,2.202,1.050,1.359$, and 1.624 , respectively. This large variation may be partly due to the variability of the intercept points at $z=0$ when the regression lines approach parallel to the axis of $z=0$.

In Figure 5, relative perceived bar length was replotted as a function of the ratio of the intermediate bar length

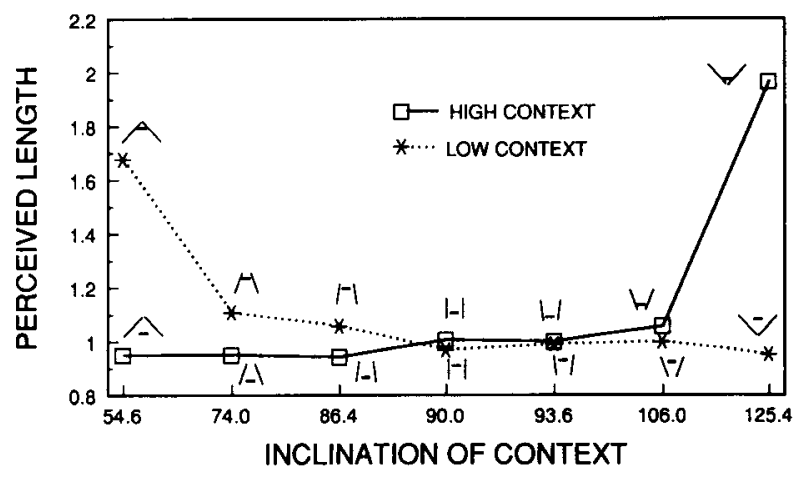

Figure 4. Perceived length of the bar as a function of the inclination of the context lines. The data are averaged for the $\mathbf{5}$ successful birds.

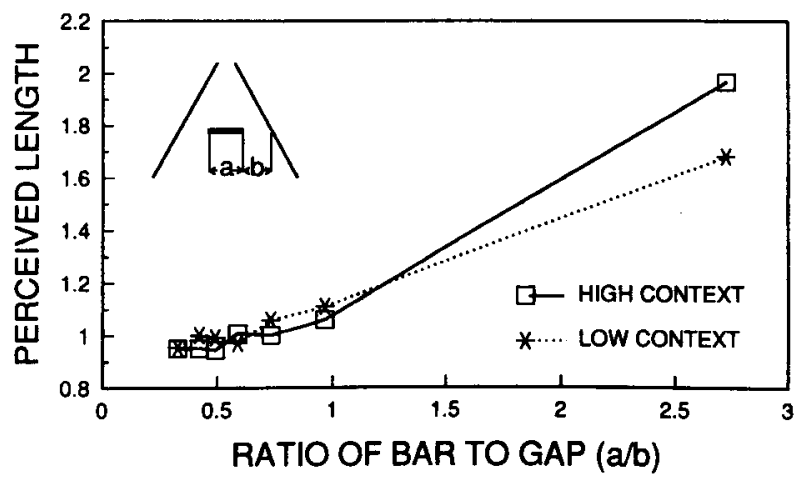

Figure 5. Perceived length of the bar as a function of the ratio of bar length to the gap between the end of the bar and context lines (a/b of the pattern in the graph). The data are averaged for the 5 successful birds. 
$(4.8 \mathrm{~mm})$ to the gap between the end of the bar and the context lines (see the drawing in the graph). The reason for doing this is that the data in Figures 3 and 4 suggest that the common feature affecting perceived length is how close the ends of the bars are to the context lines. If so, then replotting the data in terms of this ratio should reveal similarly sloped functions for both high and low contexts. The solid line with squares represents the high context condition, and the dotted line with asterisks represents the low context condition. In both conditions, perceived lengths of bars almost linearly increased as a function of the ratio of bar to gap.

A two-way analysis of variance on the data shown in Figure 5 was conducted with context location (high and low) and ratio of bar length to gap (context inclination) as fixed factors and subjects as the error term. The main effect of the ratio was highly significant $[F 6,56)=14.97$, $p<.001]$. However, the main effect of context location was not $[F(1,56)=0.06]$, nor was there a significant interaction $[F(6,56)=0.60]$. These results suggest that perceived length of bars was determined by the inclination of the context lines and that the orientation of those lines (converging upward or downward) had little effect.

\section{DISCUSSION}

Five out of 6 birds showed a consistent tendency to report bar length "long" more often when the bar was near the apex of the converging context lines (Figure 2). This replicates our earlier findings (Fujita et al., 1991) and strengthens our conclusion that pigeons perceive the Ponzo illusion. Furthermore, the illusion occurred similarly without regard to the vertical orientation of the context lines (Figure 4). Also, we found that the pigeons' choice of "long" and "short" locations correlated with the ratio of bar length to the gap between the end of the bar and the context line (bottom panel of Figure 3). The perceptual length of bars averaged for the 5 birds showing the effect changed almost linearly as a function of this ratio (Figure 5), and this relationship was similar whether the context lines converged upward or downward (Figures 4 and 5). It should also be noted that these results are unlikely to be a consequence of simple "gap" discrimination because, as was shown in the top graph of Figure 3, the gap itself could not predict the pigeons' choice of "long" and "short" locations. Apparently, the birds were responding on the basis of the perceived length of the bar.

Data on humans also suggest that the distance between the stimulus bar and the context lines is the important determinant of the Ponzo illusion. A basis for this suggestion is that the Ponzo illusion develops without the oblique context lines; for example, enclosing the test bar in staircase parallel lines or between separate dots (see Figure 6) is sufficient to produce the illusion (e.g. , Coren \& Girgus, 1978; Yamagami, 1978). Fisher $(1969,1973)$ suggested that the magnitude of illusion is determined by the gap between the stimulus bars and context lines. The present results are consistent with this account, but for a more

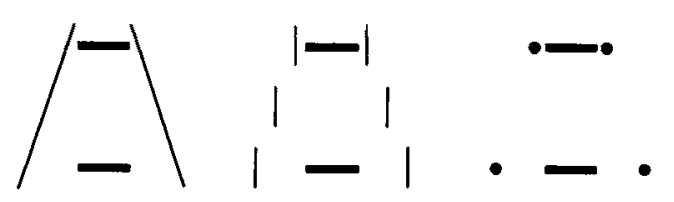

Figure 6. Typical Ponzo figures and variants that have staircase parallel lines or separate dots instead of converging line contexts.

complete test it would be necessary to test the pigeons' performances with variations in the ratio of bar to gap independent of the type and the inclination of context.

The magnitude of the illusion did not differ as a function of whether the context lines converged upward or downward. This result is akin to our previous finding that additional perspective lines do not enhance the Ponzo illusion in pigeons (Fujita et al., 1991, Experiment 3). For humans, by contrast, upright figures are a little more powerful in inducing the illusion than are horizontal or inverted figures, and this differential effect is amplified when strong perspective cues are added. Also, in humans, culture and level of education affect the magnitude of the Ponzo illusion (Brislin, 1974; Brislin \& Keating, 1976; Kilbride \& Leibowitz, 1975; Predebon, 1984; Pressey, 1974a; Quina \& Pollack, 1972; Smith, 1973; Wagner, 1977). Such findings suggest that higher cognitive processes enhance the Ponzo illusion in humans.

Gregory (1963) proposed that unconscious processing of perspective from line drawings may be the fundamental factor producing many visual illusions, including the Ponzo illusion. For example, humans routinely overestimate the size of the items that appear to be far away (like the bar near the apex of the converging lines) in accordance with size constancy. However, other findings opposed to this perspective account (Fineman \& Carlson, 1973; Humphrey \& Morgan, 1965; Newman \& Newman, 1974) indicate that such higher processes do not appear to be truly fundamental.

Taken together, the results from pigeons and humans suggest that the Ponzo illusion is attributable in large part to basic perceptual processes that may be similar in the two species, but that in humans the extent of the illusion is modulated by additional perceptual or cognitive processes.

\section{REFERENCES}

Brisuin, R. W. (1974). The Ponzo illusion: Additional cues, age, orientation, and culture. Joumal of Cross Cultural Psychology, 5, 139-161. Brislin, R. W., \& Keating, C. F. (1976). Cultural differences in the perception of a three-dimensional Ponzo illusion. Journal of Cross Cultural Psychology, 7, 397-412.

Coren, S., \& Girgus, J. S. (1978). Seeing is deceiving: The psychology of visual illusions. Hillsdale, NJ: Erlbaum.

Fineman, M. B., Carlson, J. (1973). A comparison of the Ponzo illusion with a textural analogue. Perception \& Psychophysics, 14, 31-33.

FISHER, G. H. (1969). Towards a new explanation for the geometrical illusions: $I$. The properties of contours which induce illusory distortion. British Journal of Psychology, 60, 179-185. 
FISHER, G. H. (1973). Towards a new explanation for the geometrical illusions: II. Apparent depth or contour proximity? British Journal of Psychology, 64, 607-621.

Fujita, K., Blough, D. S., \& Blough, P. M. (1991). Pigeons see the Ponzo illusion. Animal Learning \& Behavior, 19, 283-293.

GREGoRY, R. L. (1963). Distortion of visual space as inappropriate constancy scaling. Nature, 199, 678-680.

Humphrey, N. K., \& Morgan, M. J. (1965). Constancy and the geometric illusions. Nature, 206, 744-745.

Kilbride, P. L., \& Leibowitz, H. W. (1975). Factors affecting the magnitude of the Ponzo perspective illusion among the Baganda. Perception \& Psychophysics, 17, 543-548.

Leibowitz, H., Brislin, R., Perlmutter, L., \& Hennessy, R. (1969). Ponzo perspective illusions as a manifestation of space perception. Science, 166, 1174-1176.

Newman, C. V., \& Newman, B. M. (1974). The Ponzo illusion in pictures with and without suggested depth. American Journal of Psychology, 87, 511-516.

Predebon, J. (1984). Age trends in the Mueller-Lyer and Ponzo illusions. British Journal of Developmental Psychology, 3, 99-103.

Pressey, A. W. (1974a). Age changes in the Ponzo and filled-space illusions. Perception \& Psychophysics, 15, 315-319.
Pressey, A. W. (1974b). Measuring the Ponzo illusion with the method of production. Behavior Research Methods \& Instrumentation, 6, 424-426.

Pressey, A. W., Butchard, N., \& Scrivner, L. (1971). Assimilation theory and the Ponzo illusion: Quantitative predictions. Canadian Journal of Psychology, 25, 486-497.

Quina, K., \& Pollack, R. H. (1972). Effects of test line position and age on the magnitude of the Ponzo illusion. Perception \& Psychophysics, 12, 253-256.

SMITH, T. (1973). The susceptibility of Xhosa groups to a perspective illusion. Journal of Social Psychology, 90, 331-332.

WaGner, D. A. (1977). Ontogeny of the Ponzo illusion: Effects of age, schooling, and environment. International Journal of Psychology, 12, 161-176.

YamaGami, A. (1978). Two kinds of apparent size distortion in the Ponzo illusion. The Japanese Journal of Psychology, 49, 273-279. (In Japanese with English summary)

(Manuscript received January 31, 1992; revision accepted for publication June 1,1992 .)

\section{Forthcoming Articles}

The following is a list of forthcoming Animal Learning \& Behavior articles that are currently in press. They are given in approximate order of acceptance. Each entry includes the name and address of the author with whom to communicate for further prepublication information.

"Reinforced variation and selection"' by A. Neuringer (Dept. of Psychology, Reed College, Portland, OR 97202)

"Classical conditioning of jaw movements in the pigeon: Acquisition and response topography" by M. Remy \& H.P. Zeigler (H.P.Z., Dept. of Psychology, Hunter College, 695 Park Ave., New York, NY 10021)

"Coding of feature and no-feature events by pigeons performing a delayed conditional discrimination"' by L.M. Sherburne \& T.R. Zentall (L.M.S., Dept. of Psychology, Univ. of Kentucky, Lexington, KY 40506)

" One bottle too many? Method of testing determines the detection of overshadowing and retention of taste aversions' by W.R. Batsell, Jr., \& M.R. Best (W.R.B., Dept. of Psychology, Southern Methodist Univ., Dallas, TX 75275)

"The influence of brief stimuli uncorrelated with reinforcement on choice between variable-ratio schedules" by P. Reed, V. Szczudlo, A. Willis, \& G. Hall (P.R., Dept. of Psychology, Univ. College London, Gower Street, London WC1 6BT, U.K.)

"Attenuation by contextual cues of retroactive interference of a conditional discrimination in rats" by W.A. Rodriguez, L.S. Borberly, \& R.S. Garcia (W.A.R., Dept. of Psychology, 3210 Tolman Hall, Univ. of California, Berkeley, CA 94720)

“Cross-stimulus transfer of timing in pigeons"' by D.S. Grant \& T.C. Robinson (D.S.G., Dept. of Psychology, Univ. of Alberta, Edmonton, AB, Canada T6G 2E9)

"Aversively motivated changes in meal patterns of rats in a closed economy: The effects of shock density"' by F.J. Helmstetter \& M.S. Fanselow (F.J.H., Dept. of Psychology, Univ. of Wisconsin, P.O. Box 413, Milwaukee, WI 53201)
"Associative properties of a conditioned inhibitor as a function of age in kittens"' by N.K. Dess \& S.S. Soltysik (N.K.D., Dept. of Psychology, Occidental College, 1600 Campus Rd., Los Angeles, CA 90041)

"Summation and transfer of negative occasion setting" by P.C. Holland \& J.R. Morell (P.C.H., Dept. of Experimental Psych., Duke Univ., Durham, NC 27706)

"Representation strength in pigeon short-term memory: Effect of delay training"' by J.N. Steirn, T.R. Zentall, \& L.M. Sherburne (J.N.S., Dept. of Psych., Landrum Box 8041, Georgia Southern Univ., Statesboro, GA 30460)

"Memory codes for temporal and nontemporal samples in manyto-one matching by pigeons"' by A. Santi, S. Bridson, \& M. Ducharme (A.S., Dept. of Psych., Wilfrid Laurier Univ., Waterloo, ONT N2L 3C5, Canada)

"Preservation of response-outcome associations through extinction" by R.A. Rescorla (Dept. of Psychology, Univ. of Pennsylvania, 3815 Walnut St., Philadelphia, PA 19104)

"Search behavior of dogs (Canis familiaris) in invisible displacement problems"' by S. Gagnon \& F.Y. Dore (S.G., Dept. of Psychologie, Univ. du Quebec a Montreal, CP 8888, Succ A, Montreal, Quebec, Canada H3C 3P8)

"Choice in honeybees as a function of the probability of reward" by M.E. Fischer, P.A. Couvillon, \& M.E. Bitterman (M.E.B., Békésy Laboratory of Neurobiology, 1993 EastWest Rd., Honolulu, HI 96822)

"Interference among modulators" by R.A. Rescorla (Dept. of Psychology, Univ. of Pennsylvania, 3815 Walnut Street, Philadelphia, PA 19104) 